\title{
ESTUDO TERMOGRAVIMÉTRICO DO PROCESSO DE SORÇÃO DE METAIS PESADOS POR RESÍDUOS SÓLIDOS ORGÂNICOS
}

\author{
THERMOGRAVIMETRIC STUDY OF THE SORPTION PROCESS OF HEAVY \\ METALS IN ORGANIC SOLID WASTE
}

\section{ALDRE Jorge Morais BARROS}

Químico Industrial pela Universidade Estadual da Paraíba. Mestre em Engenharia Sanitária pela Universidade Federal de Campina Grande. Doutor em Química pelo Departamento de Química da Universidade Federal da Paraíba
\end{abstract}

\begin{abstract}
SHIVA PRASAD
Bacharel. Mestre e Doutor em Química pela University of Rajasthan, Jaipur/Índia. Professor Titular do Departamento de Engenharia Química da Universidade Federal de Campina Grande, PB
\end{abstract}

\section{VALDERI DUARTE LEITE}

Engenheiro Químico e Mestre em Engenharia Sanitária pela Universidade Federal da Paraíba. Doutor em Hidráulica e Saneamento pela Escola de Engenharia de São Carlos da Universidade de São Paulo. Professor Titular do

Departamento de Química da Universidade Estadual da Paraíba

\begin{abstract}
Antonio Gouveia Souza
Licenciado em Química pela Universidade Estadual da Paraíba. Bacharel em Química pela Universidade Federal da Paraíba. Doutor em Química pela Universidade de Campinas. Professor Adjunto do Departamento de Química da Universidade Federal da Paraíba
\end{abstract}

Recebido: 08/08/05 Aprovado: 30/05/06

\section{RESUMO}

A poluição de uma bacia hidrográfica pode causar alterações das propriedades físicas, químicas e biológicas da massa de água, comprometendo o seu uso para o consumo humano e para outros tipos de atividades, dependendo do grau de poluição ou contaminação. Os métodos de tratamentos convencionais de efluentes contendo metais pesados produzem quantidades significativas de subprodutos com características recalcitrantes, além de serem técnicas onerosas e de reprodutibilidade baixa. A busca por soluçôes mais baratas e definitivas levou ao desenvolvimento de tecnologias limpas, dentre estas tecnologias destaca-se o processo de sorção que baseia-se na utilização de substâncias absorventes desses elementos químicos. Este trabalho teve como objetivo avaliar o processo de sorção de metais pesados pelos resíduos sólidos orgânicos utilizando-se das técnicas de termogravimetria. Os resultados obtidos demonstraram que os perfis das curvas da termogravimetria dos resíduos sólidos orgânicos apresentam modificaçōes significativas nas características físicas e químicas, quando submetidas ao processo de sorção dos metais pesados.

PALAVRAS-CHAVE: Metais pesados, resíduos sólidos orgânicos, sorção, análise térmica.

\begin{abstract}
Pollution of a hydrographic basin can cause alterations in physical, chemical and biological properties of water mass, committing its use for the human consumption and for other types of activities, depending on the contamination or pollution level. The conventional treatment methods for effluents containing heavy metals produce significant amounts of byproducts with recalcitrant characteristics; besides the techniques being onerous their reproducibility is low. The search for cheaper and definitive solutions led to the development of clean technologies, among these technologies the sorption process based on utilization of absorbent substances for these elements can be pointed out. This work has the objective of evaluating the sorption process of heavy metals by organic solid waste utilizing the thermogravimetric technique. The results obtained demonstrated that the profiles of the thermogravimetric curves of the organic solid waste presented significant modifications in the physical and chemical characteristics when submitted to the heavy metal sorption process.
\end{abstract}

KEYWORDS: Heavy metals, organic solid waste, sorption, thermal analysis.

\section{INTRODUÇÃO}

Os metais pesados reduzem a capacidade autodepurativa das águas, devido à ação tóxica exercida por estes elementos sobre os microrganismos responsáveis pela depuração natural em sistemas aquáticos (Branco, 1986). Este tipo de poluição do meio aquático pode causar alterações das característi- cas físicas (turbidez, cor, temperatura, viscosidade, tensão superficial), químicas (DQO, pH, acidez, alcalinidade, força iônica, OD, grau de toxicidade, nutrientes) ou biológicos (espécies de 
fitoplâncton e zooplâncton), comprometendo a qualidade da água para o consumo humano (Clarisse et al, 1999), por exemplo, uma elevada concentração de oxigênio na água nem sempre significa um indício de condições aeróbias saudáveis, podendo ser promovido pelo envenenamento com metais pesados, devido à formação de óxidos metálicos de uma determinada massa da água (Fellenberg, 1980).

Os metais pesados, dentre os vários poluentes existentes, têm recebido atenção especial, uma vez que alguns são extremamentes tóxicos, quando estão acima das concentraçóes permissíveis para uma grande variedade de organismos, mesmo em concentrações em torno de mili ou microgramas. Com o aumento dos níveis de contaminação do meio ambiente por metais pesados, tem-se tornado mais econômico e sensato tratar os esgotos e despejos industriais com verdadeira eficácia, ao invés de tentar sanar os efeitos danosos produzidos pelos efluentes não tratados. Os tratamentos convencionais dos efluentes contaminados com metais pesados envolvem processos físico-químicos de floculação e/ou precipitação, eletrólise e cristalização. Contudo, estes processos são caros e produzem novos resíduos, que muitas vezes se utilizam de substâncias tóxicas como agentes complexantes, e que inviabilizam a possibilidade da reciclagem ou recuperação destes metais, não propiciando, assim, uma solução definitiva e ambientalmente correta da destinação destes metais.

A busca por soluções menos onerosas e definitivas levou ao desenvolvimento de metodologias baseadas na utilização de substratos orgânicos naturais na remoção dos metais pesados num processo denominado "sorção", que é caracterizado pelo uso de sistemas de reatores com pequenos volumes e altas concentraçôes de substratos orgânicos presente em seu interior (Metcalf e Eddy, 1991; Pinto et al, 2001), podendo ser constituído por matéria orgânica viva (bactérias, fungos, etc) ou matéria orgânica morta (casca, folhas, etc).

O processo de sorção pode ser definido como sendo a concentração ou acumulação de íons ou moléculas sobre uma superfície absorvente, devido à ação de dois fenômenos simultâneos bastante distintos. O primeiro é a adsorção, onde as moléculas de um soluto são atraídas para sítios vagos na superfície de um substrato, fixando-se nestes locais em virtude da ação de forças físicas ou de ligações químicas (Valdman e Leite, 2000; Sawyer et al,1994). A adsorção física (fisissorção) é relativamente não específica, haja vista ocorrer devido à ação de pequenas forças intermoleculares (por exemplo, interação de dispersão, interação dipolo-dipolo) entre as moléculas, logo, a molécula do adsorvato não está presa à superfície do absorvente podendo se mover pelo material fixador, sendo, geralmente, reversível (Atkins, 1999).

Por outro lado, adsorção química (quimissorção) é o resultado da formação de ligações químicas. Normalmente, o adsorvato forma uma camada sobre a superfície do absorvente, e as moléculas não são consideradas livres para moverse de um local da superfície para outro. Quando a superfície é coberta por uma capa monomolecular do adsorvato, a capacidade do absorvente é essencialmente eficiente (Sawyer et al,1994; Valdman e Leite, 2000). Quando a sorção dos metais dissolvidos está baseada na atividade química da biomassa microbiana ou do resíduo vegetal morto é denominada de biossorção, sendo o fundamento para uma nova tecnologia na remoção e recuperação desses metais (Barros Jr. et al, 2002).

A biomassa microbiana inativa e/ou morta pode servir como uma base para o desenvolvimento de materiais biossorventes que podem ser usados para captura e recuperação de metais pesados. Desde que estes novos biossorventes possam ser regenerados, reutilizados, seletivos, eficientes, baratos e competitivos com produtos artificiais, e que apresentem a aplicação potencial no controle ambiental dos metais e nas operaçôes de recuperação metálica (Volesky, 1990). Dentre estes biossorventes o resíduo sólido orgânico é um sedimento constituído de sais minerais, compostos orgânicos e inorgânicos, que segundo Arican et al (2002), a forte atração dos metais por este tipo de substrato ocorre devido à formação de ácidos húmicos oriundos da matéria orgânica. Estas substâncias são ricas em grupos funcionais com cargas negativas, tais como ácidos carboxílicos, hidroxilas fenólicas e alcoólicas, que são os sítios de complexação dos metais em solução.

A análise térmica é um conjunto de técnicas que permite medir as mudanças de uma propriedade física ou química de uma substância ou material em função da temperatura e/ou tempo, enquanto a substância é submetida a uma programação controlada de temperatura. Os resultados obtidos pela análise térmica de uma amostra são representados graficamente em uma curva obtida durante o processo de termodecomposição de uma amostra bruta, tornando desnecessária a necessidade de pré-tratamento ou abertura da mesma, que pode ser aplicada em estudos de: (1) decomposição térmica; (2) determinação de umidade, de produtos voláteis, de resíduos e de teor de cinzas; (3) oxidação térmica; (4) cinética de reação de cura e cristalização; (5) diagrama de fases; (6) determinação do calor específico; (7) determinação de transição vítrea, de fusão; etc. Dentre as técnicas de análise térmica, a termogravimetria (TG) baseia-se no estudo da variação de massa de uma dada amostra, resultante da transformação física (sublimação, evaporação, condensação) ou química (degradaçãa, decomposição, oxidação) em função do tempo e da temperatura (Souza et al,2004; Mothé e Azevedo, 2002; Hatakeyama e Quinn, 1997).

Este trabalho teve como objetivo, estudar as modificaçôes das características físicas e químicas do resíduo sólido orgânico (RSO) submetido ao processo de sorção com espécies metálicas conhecidas, utilizando-se das técnicas termogravimétricas e espectroscopia de infravermelho.

\section{MATERIAL E MÉTODOS}

\section{Sistema experimental}

O sistema experimental para realização do estudo de sorção dos metais pesados $\left(\mathrm{Ni}^{2+}, \mathrm{Cr}^{3+}, \mathrm{Co}^{3+}, \mathrm{Cu}^{2+}\right.$ e $\mathrm{Cd}^{2+}$ ) foi instalado e monitorado no Laboratório de Saneamento Ambiental da Universidade Estadual da Paraíba (UEPB) localizado na estação experimental de tratamento biológico de esgoto sanitário (EXTRABES), localizado no bairro Tambor da cidade de Campina Grande, Paraíba (7013'11" $\mathrm{S}, 35^{\circ} 52^{\prime} 31^{\prime \prime} \mathrm{O}, 550 \mathrm{~m}$ acima do nível do mar), nordeste do Brasil.

O resíduo sólido orgânico (RSO) utilizado no sistema experimental constou de amostras pontuais de resíduos sólidos orgânicos coletados na central de abastecimento de Campina Grande da empresa paraibana de serviços agrícolas (EMPASA), seguido pelo acondicionamento em uma caixa de fibra de vidro 
de seção retangular para secagem ao ar. Este resíduo era revolvido diariamente para facilitar a secagem, que ocorreu num período de 12 dias. O RSO seco foi triturado e passado numa peneira de malha de 50 mesh e acondicionado em sacos plásticos.

Para promover a sorção entre o metal e RSO foram utilizados seis reatores de batelada acondicionados em um agitador Jar Test modelo JT-103 fabricado pela QUIMIS (Figura 1). Em cada um dos cinco reatores foi adicionado um volume de um litro de solução metálica cuja concentração foi de 50,0 mg. $\mathrm{L}^{-1}$ de cada íon metálico usado nesta pesquisa, o sexto reator foi carregado com um litro de água deionizada como branco do sistema em estudo. Todos os reatores receberam uma massa de 5,0 g de RSO quantitativamente pesado e diluído em água deionizada numa proporção de 1:200, segundo recomendaçóes de Volesky (1989). O tempo de reação estudado neste trabalho foi $24 \mathrm{~h}$ para os seis reatores, sendo o experimento realizado em triplicata, procedido pela filtração do mistura sólido-líquido e secagem a $105^{\circ} \mathrm{C}$ em estufa por $24 \mathrm{~h}$ para eliminação do excesso de umidade.

\section{Procedimentos analíticos}

As medidas das concentrações de sólidos totais e frações (fixos e voláteis), carbono orgânico total (COT), nitrogênio total (NTK) e demanda química de oxigênio (DQO) das amostras sólidas obtidas antes e após processo de sorção dos metais foram determinados pela metodologia preconizada pelo APHA et al (1995).

Os dados da análise térmica (TG e DTG) das amostras foram obtidos através de um sistema de termobalan-

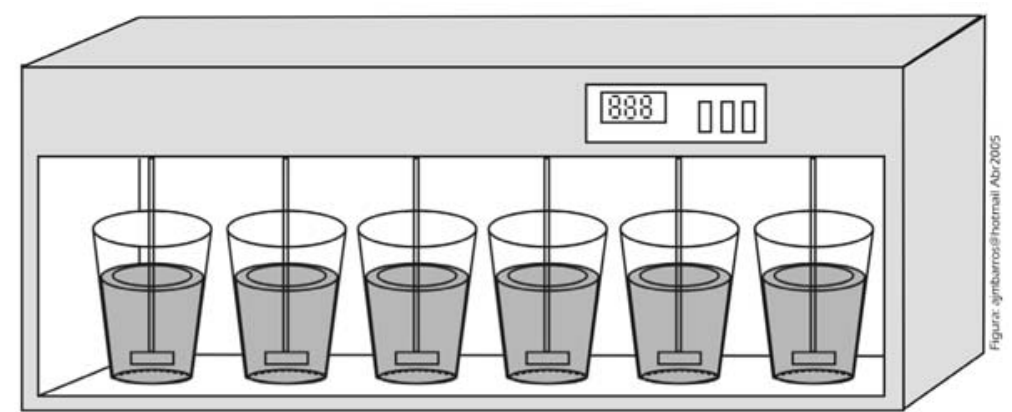

Figura I - Esquema do sistema experimental de sorção dos metais

ça, marca SHIMADZU, em atmosfera de ar sintético com vazão constante de $20 \mathrm{~mL} \cdot \mathrm{min}^{-1}$, utilizando-se um cadinho de alumina para acondicionamento das amostras, com razão de aquecimento de $10{ }^{\circ} \mathrm{C} \cdot \mathrm{min}^{-1}$. Foram tomadas amostras com massa média de 5,4 \pm 0,13 mg, após a passagem das amostras bruta em uma peneira de 200 mesh, para uma melhor reprodutibilidade e uniformidade dos resultados. Os dados foram registrados e sofreram um tratamento matemático no software TASYS for Windows fornecido pelo fabricante da termobalança.

A análise de espectroscopia vibracional de absorção na região infravermelho nas amostras de RSO e RSO + metal (RM) foram obtidos em pastilhas de $\mathrm{KBr}$, prensadas sob vácuo. Os espectros absorção na região do infravermelho foram registrados utilizando-se um espectrofotômetro marca BOMEM, modelo MB-100M na regiāo compreendida entre 4000 e $400 \mathrm{~cm}^{-1}$.

\section{RESULTADOS E DISCUSSÃO}

$\mathrm{Na}$ Tabela 1 estão sendo apresentados os dados referentes à umidade, sólidos totais (ST) e suas fraçôes fixas (STF) e voláteis (STV), carbono or- gânico total (COT), nitrogênio total (NTK) e demanda química de oxigênio (DQO).

Os resultados obtidos demonstraram significativas alteraçôes nas concentrações originais dos resíduos sólidos orgânicos (RSO) submetidos ao processo de sorção dos metais $\left(\mathrm{Ni}^{2+}\right.$, $\mathrm{Cr}^{3+}, \mathrm{Co}^{3+}, \mathrm{Cu}^{2+}$ e $\left.\mathrm{Cd}^{2+}\right)$. A umidade do RSO apresentou uma redução significativa após a sorção dos metais pelo RSO com uma redução média em torno de $2,6 \%$, podendo ser atribuída a mineralização do biossólido, ocasionando à diminuição dos sítios hidrolisados da superfície sólida.

As concentrações dos sólidos totais das amostras RM proporcionaram um aumento em relação ao RSO com a concentração de $87,1 \%$, para as amostras que sorveram os metais as concentraçōes médias foram de 89,6\%, os valores extremos (mínimo e máximo) foram obtidos nas amostras RCr e RCo como 89,2 e $90,2 \%$, respectivamente. Os sólidos totais fixos das amostras RM apresentaram valores variando entre 20,6 e 23,6\% para as amostras $\mathrm{RCo}$ e $\mathrm{RCu}$, correspondentemente, sendo estes dados menores que 24,8\% da amostra RSO.

Os sólidos totais voláteis das amostras RM apresentaram resultados

Tabela I - Valores médios da caracterização química do RSO e do RM

\begin{tabular}{cccccccc}
\hline Amostras & $\begin{array}{c}\text { Umidade } \\
(\%)^{\mathrm{a}}\end{array}$ & $\begin{array}{c}\text { ST } \\
(\%)\end{array}$ & $\begin{array}{c}\text { STF } \\
(\%)\end{array}$ & $\begin{array}{c}\text { STV } \\
(\%)\end{array}$ & $\begin{array}{c}\text { COT } \\
(\%)\end{array}$ & $\begin{array}{c}\text { NTK } \\
(\%)\end{array}$ & $\begin{array}{c}\text { DQO } \\
(\%)\end{array}$ \\
\hline RSO & 12,9 & 87,1 & 24,8 & 75,2 & 41,8 & 22,4 & 76,4 \\
RCr & 10,8 & 89,2 & 21,7 & 78,3 & 43,5 & 23,0 & 33,0 \\
RNi & 10,7 & 89,3 & 20,8 & 79,2 & 44,0 & 20,2 & 71,9 \\
RCd & 10,2 & 89,8 & 22,9 & 77,1 & 42,8 & 26,7 & 57,4 \\
RCu & 10,3 & 89,7 & 23,6 & 76,4 & 42,5 & 20,7 & 83,4 \\
RCo & 9,8 & 90,2 & 20,6 & 79,4 & 44,1 & 20,3 & 43,0 \\
\hline
\end{tabular}

${ }^{a}$ Porcentagem em peso seco 
entre 76,4 e 79,4\% das amostras $\mathrm{RCu}$ e RCo, respectivamente. Estes valores foram maiores que $75,2 \%$ da amostra RSO, este aumento pode ter ocorrido pela formação de grupos orgânicos carbonilados, que proporcionam reaçóes entre absorvente e os íons metálicos. Esta observação pode ser reforçada pelos valores das concentraçôes de COT variaram entre 42,5 a $44,1 \%$ para as amostras RCu e RCo, correspondentemente, com concentraçóes maiores que a amostra RSO que continha um COT de $41,8 \%$.

As concentrações de nitrogênio variaram entre 20,2 a 26,7\% para as amostras RNi e RCd, respectivamente. A amostra RSO apresentou uma concentração de $22,4 \%$. A presença de nitrogênio foi confirmado pela observação de bandas de absorção v $(\mathrm{N}-\mathrm{H})$ em torno $3350 \mathrm{~cm}^{-1}$ nos espectros de infravermelho das amostras de RCr, RCd e RCu (Tabela 2 e Figura 2), em concordância com maiores concentrações de nitrogênio encontradas nestas amostras (Tabela
1), correspondentemente. A banda de absorção v(N-H) não pode ser visualizada na amostra RSO, devido ao maior teor de umidade (Tabela 1), que ocasionou o encobrimento do estiramento de $\mathrm{v}(\mathrm{N}-\mathrm{H})$ desta amostra devido à presença de uma banda larga e intensa atribuída a v $(\mathrm{O}-\mathrm{H})$ de grupos carbonilados ou da própria molécula de água $(\mathrm{H}-\mathrm{O}-\mathrm{H})$.

$\mathrm{Na}$ Tabela 2 e Figura 2 estão sendo apresentados as principais bandas de absorção e espectros das amostras na região de infravermelho, respectivamente. A Figura 2a está apresentando o espectro da amostra RSO. As características observadas denotam um comportamento semelhante ao perfil de compostos carbonilados, devido à presença de bandas de vibração $\mathrm{v}(\mathrm{O}-\mathrm{H})$

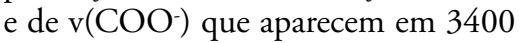
a $2400 \mathrm{~cm}^{-1}$ e de 1300 a $1080 \mathrm{~cm}^{-1}$, respectivamente. Enquanto, na Figura $2 \mathrm{~b}$ estão apresentados os espectros das amostras que sorveram os metais, que apresentaram a presença de bandas características de compostos químicos ligados a grupos orgânicos oxigenados (Pavia et al, 1996).

Analisando os espectros das amostras, pode-se observar a presença de bandas de estiramento assimétricos e simétricos do grupo $\mathrm{RCHOO}$ em torno de 1760 a $1690 \mathrm{~cm}^{-1}$ e de $1300 \mathrm{a}$ $1080 \mathrm{~cm}^{-1}$, decorrente da formação de compostos carbonilados coordenados, e que foi confirmado pela presença da banda larga de absorção do grupo $\mathrm{v}(\mathrm{O}-\mathrm{H})$ nas seis amostras entre $3500 \mathrm{a}$ $2500 \mathrm{~cm}^{-1}$, observou-se também bandas vibracionais características de v $(\mathrm{C}-\mathrm{H})$ e $\mathrm{v}\left(\mathrm{CH}_{3}\right)$ em 2960 a $2850 \mathrm{~cm}^{-1}$, e de 1470 a $1350 \mathrm{~cm}^{-1}$, respectivamente.

As amostras $\mathrm{RCr}$, RCd e $\mathrm{RCu}$ apresentaram bandas fortes de absorção do grupo nitrogenados na forma de aminas de $\mathrm{v}(\mathrm{N}-\mathrm{H})$ e v $(\mathrm{C}-\mathrm{N})$ em torno de 3500 a $3300 \mathrm{~cm}^{-1}$, e de 1360 a $1180 \mathrm{~cm}^{-1}$, correspondentemente, estas bandas foram confirmadas pela análise de NTK (Tabela 1) das amostras, o não aparecimento dessas bandas nas amostras RSO, $\mathrm{RNi}$ e RCo ocorreu devido à banda
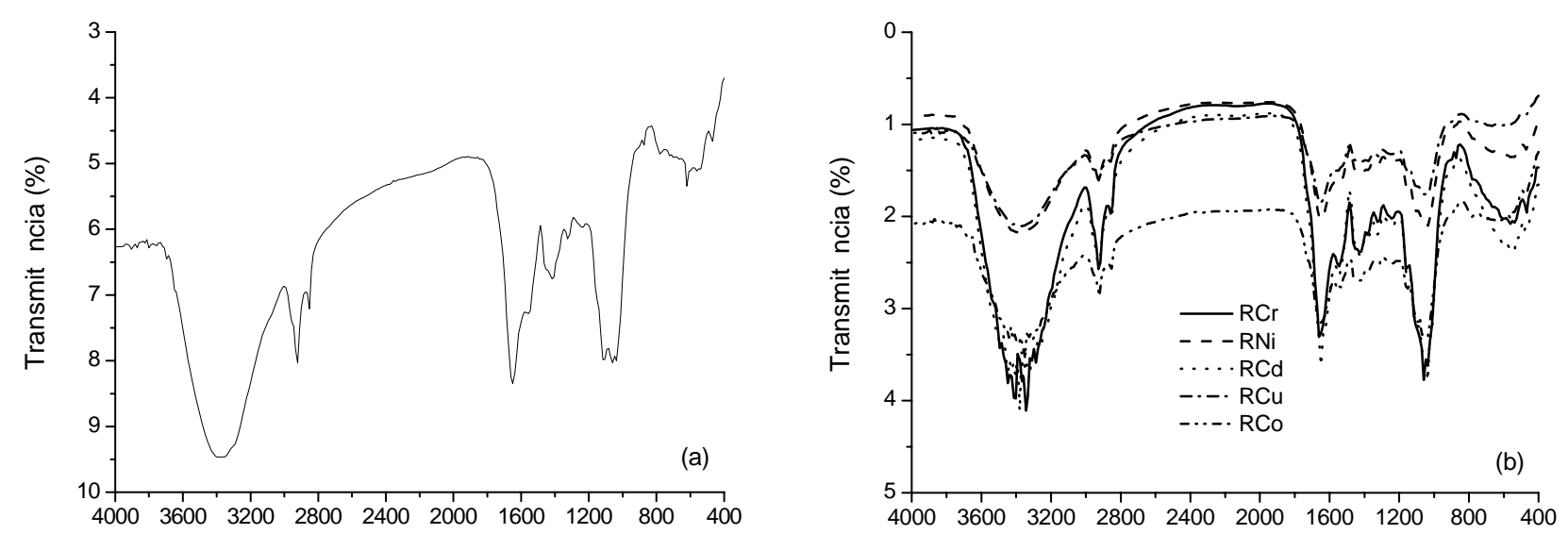

Figura 2 - Espectros de absorção na região do infravermelho para as amostras:

(a) RSO; (b) RCr, RNi, RCd, RCu e RCo.

Tabela 2 - Principais bandas de absorção no infravermelho das amostras RSO e RM

\begin{tabular}{ccccccc}
\hline Amostras & \multicolumn{5}{c}{ Números de ondas $\left(\mathrm{cm}^{-1}\right)$} \\
& $\mathrm{v}(\mathrm{O}-\mathrm{H})$ & $\mathrm{v}(\mathrm{C}-\mathrm{H})$ & $\mathrm{v}(\mathrm{N}-\mathrm{H})$ & $\mathrm{v}(\mathrm{C}=\mathrm{O})$ & $\mathrm{v}(\mathrm{C}-\mathrm{N})$ & $\mathrm{v}(\mathrm{M}-\mathrm{O})$ \\
\hline $\mathrm{RSO}$ & $3378(\mathrm{~F})^{\mathrm{a}}$ & $2922(\mathrm{~F})$ & - & $1641(\mathrm{~F})$ & - & - \\
$\mathrm{RCr}$ & $3400(\mathrm{~F})$ & $2925(\mathrm{~F})$ & $3335(\mathrm{~F})$ & $1654(\mathrm{~m})$ & $1237(\mathrm{~m})$ & $471(\mathrm{f})$ \\
$\mathrm{RNi}$ & $3375(\mathrm{~m})$ & $2925(\mathrm{~F})$ & - & $1647(\mathrm{~m})$ & $1242(\mathrm{~m})$ & $464(\mathrm{f})$ \\
$\mathrm{RCd}$ & $3400(\mathrm{~F})$ & $2916(\mathrm{~F})$ & $3383(\mathrm{~F})$ & $1654(\mathrm{~F})$ & $1295(\mathrm{~F})$ & $533(\mathrm{f})$ \\
$\mathrm{RCu}$ & $3300(\mathrm{~F})$ & $2916(\mathrm{~F})$ & $3345(\mathrm{~F})$ & $1647(\mathrm{~F})$ & $1245(\mathrm{~F})$ & $471(\mathrm{f})$ \\
$\mathrm{RCo}$ & $3375(\mathrm{~m})$ & $2925(\mathrm{~F})$ & - & $1647(\mathrm{~F})$ & $1237(\mathrm{~F})$ & $470(\mathrm{f})$ \\
\hline
\end{tabular}

aAs observaçóes em parênteses estão relacionadas à intensidade ( $\mathrm{F}$ - Forte; $\mathrm{f}$ - fraca; $\mathrm{m}$ - média) de cada banda de vibração (v) na região do infravermelho 
larga do $\mathrm{v}(\mathrm{OH})$ que encobrem bandas de menor intensidade. As bandas de estiramentos da ligação metal-oxigênio foram observadas nas amostras RM em torno de 540 a $423 \mathrm{~cm}^{-1}$, confirmando a interação entre o absorvente e os íons metais, através da formação de ligações químicas entre grupos carbonilados com os metais pesados.

Ferenc e Walków-Dziewuslka (2003) ao estudarem a formação de complexos com dois tipos de metais pesados encontraram espectros de infravermelho semelhantes aos deste trabalho, bandas de absorção no infravermelho parecidas com esta pesquisa também foram obtidos por Mallikarjuna e Venkatarama (2003) que estudaram a sorção do chumbo por um sistema inorgânico.

$\mathrm{Na}$ Figura 3 e na Tabela 3 estão sendo apresentados os dados da análise térmica (TG/DTG) das amostras RSO, RCr, RNi, RCd, RCu e RCo. Os resultados obtidos demonstraram que as temperaturas de decomposição térmica sofreram deslocamentos após a sorção dos metais por parte do biossólido usado neste trabalho. Os perfis das curvas termogravimétricas apresentaram modificações nas amostras que sorveram os metais, estas alterações puderam ser confirmadas no estudo dos perfis das curvas DTG.

Os resultados obtidos demonstraram que as temperaturas de decomposição térmica das amostras que continham metais sofreram deslocamentos nos valores observados no perfil das
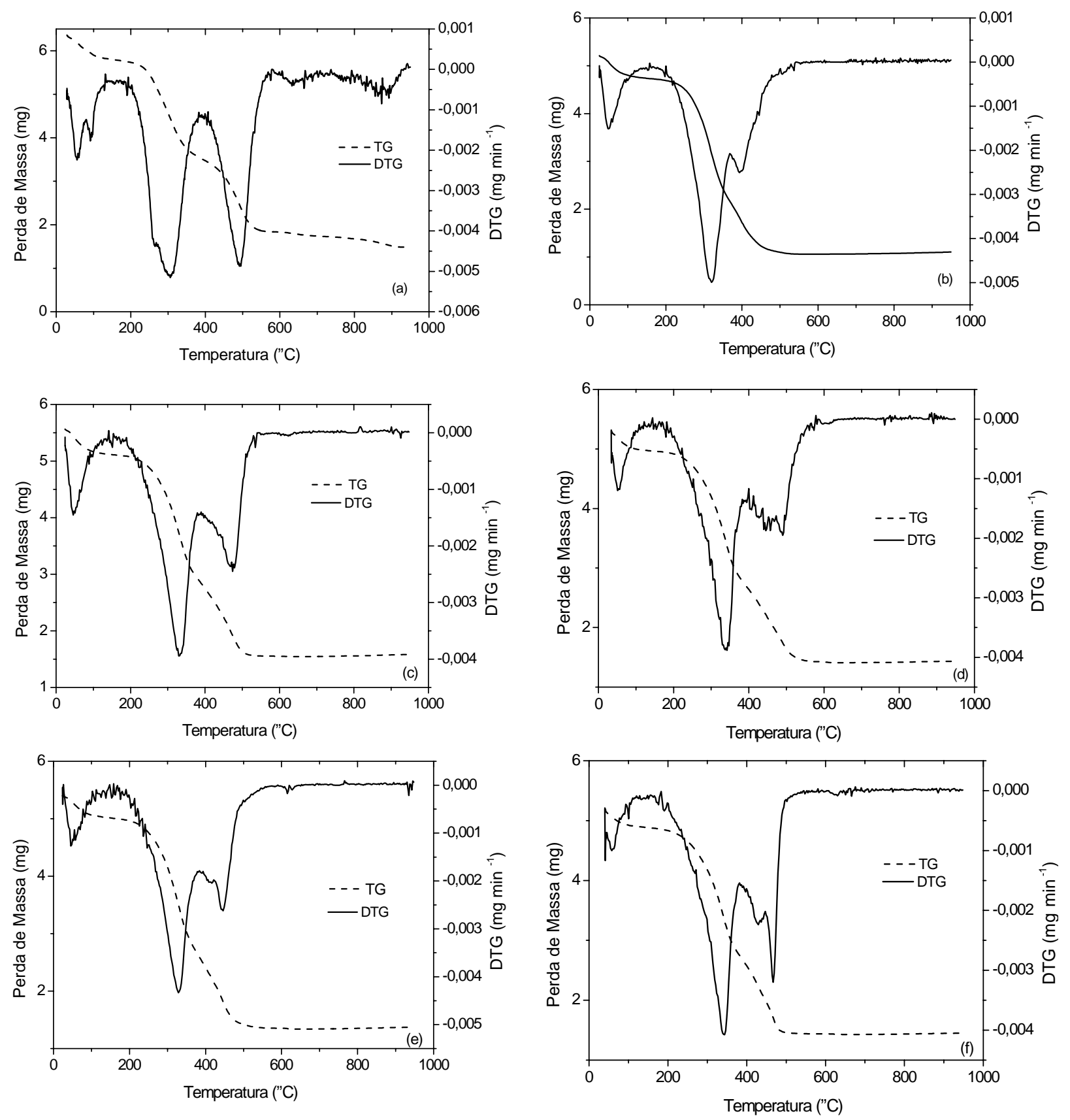

Figura 3 - Perfil termogravimétrico (TG/DTG) do: (a) RSO; (b) RCr; (c) RNi; (d) RCd; (e) RCu; (f) RCo 
curvas TG/DTG em relação à amostra RSO. As amostras que sorveram os metais apresentaram modificações significativas na decomposição do material usado como absorvente, segundo aos dados dos eventos (variação no perfil da curva) apresentados na Tabela 3 das amostras RM, em relação à amostra RSO. Esta observação foi reforçada pelas mudanças das bandas no infravermelho (Tabela 2 e Figura 2) do absorvente, quando inclui o metal em seu sistema por intermédio de interações físico-químicas. Por outro lado, as alteraçóes das concentraçóes dos parâmetros apresentados na Tabela 1 , demonstraram que existe uma tendência no aumento da fração inorgânica das amostras RM, devido à retenção do metal por parte do biossólido (Barros et al, 2004).

Observando as curvas TG/DTG (Figura 3) e os dados obtidos pela análise destas curvas, pode-se verificar a redução da umidade, que graficamente demonstrado pelo aparecimento de dois eventos na curva da amostra RSO (Figura 3a), em torno de $61{ }^{\circ} \mathrm{C}$ corresponde à perda de água, seguida por dois eventos de decomposição de matéria orgânica em torno de 296 e $481{ }^{\circ} \mathrm{C}$, respectivamente.

O primeiro evento observado nas amostras RM é caracterizado pela perda de umidade, assumindo a seguinte seqüência quantitativa na perda de massa por parte das amostras: $\mathrm{Cr}^{3+}>\mathrm{Ni}^{2+}>\mathrm{Cu}^{2+}>\mathrm{Cd}^{2+}>\mathrm{Co}^{3+}$, com destaque para $\mathrm{RCr}$ que apresentou perda de massa de 8,5\% semelhante a $\mathrm{RSO}$, o RCo foi a amostra que apresentou a menor redução mássica, em torno de 5,2\%.

Este tipo de umidade pode ser atribuída à água de constituição do biossólido, demonstrando assim que grande parte da umidade do absorvente tem fundamento na hidrólise do biossólido. Portanto, a umidade residual do absorvente é decorrente da umidade atmosférica sobre a superfície do mesmo.

No segundo evento a amostra $\mathrm{RCu}$ apresentou o maior percentual de redução mássica, com $6,1 \%$ para amostras que foram submetidas ao processo de sorção, a amostra RSO apresentou uma decomposição de 36,3\%. As outras amostras apresentaram decomposições muito menores variando entre 1,8 a $5,6 \%$, onde a presença dos metais provocou uma estabilização da matéria orgânica devido à formação ligaçôes químicas entre a superfície do sólido e o metal, onde está ligação ocorreu com grupos carbonilados, que, geralmente, decompõem-se em temperaturas em torno $200{ }^{\circ} \mathrm{C}$ (Paiva et al, 1996). Esta observação está em concordância com a análise de infravermelho realizada nas amostras em estudo.

O terceiro e quarto eventos foram onde ocorreram os maiores percentuais de perda de massa para as amostras estudadas, a amostra RCr obteve maiores valores de decomposição que foram de 47,2 e $15,8 \%$ para $3^{\circ}$ e $4^{\circ}$ evento, respectivamente, com temperaturas de decomposição em torno de 312 e $396{ }^{\circ} \mathrm{C}$. As amostras com menores decomposições foram RNi com 38\% numa temperatura de $321{ }^{\circ} \mathrm{C}$ para o terceiro evento e $\mathrm{RCu}$ com $9,2 \%$ para uma temperatura de $406^{\circ} \mathrm{C}$ no quarto evento.

No quinto evento as decomposições das amostras obtiveram um com-

Tabela 3 - Dados obtidos pela análise termogravimétrica das amostras

\begin{tabular}{|c|c|c|c|c|c|c|c|}
\hline \multirow[t]{2}{*}{ Amostra } & \multirow[t]{2}{*}{ Parâmetros } & \multicolumn{5}{|c|}{ Eventos } & \multirow{2}{*}{$\begin{array}{c}\text { Processo } \\
\text { total }\end{array}$} \\
\hline & & $1^{\circ}$ & $2^{\circ}$ & $3^{\circ}$ & $4^{\circ}$ & $5^{\circ}$ & \\
\hline \multirow[t]{3}{*}{$\mathrm{RSO}$} & $\mathrm{Ti}^{*}\left({ }^{\circ} \mathrm{C}\right)$ & 29,0 & 144,0 & 395,0 & 588,0 & 741,0 & 29,0 \\
\hline & $\mathrm{Tp}^{* *}\left({ }^{\circ} \mathrm{C}\right)$ & 68,0 & 296,0 & 481,0 & 647,0 & 856,0 & 336,0 \\
\hline & Perda de massa (\%) & 8,4 & 36,3 & 26,0 & 1,9 & 3,6 & 76,31 \\
\hline \multirow[t]{3}{*}{$\mathrm{RCr}$} & $\mathrm{Ti}\left({ }^{\circ} \mathrm{C}\right)$ & 27,0 & 134,0 & 219,0 & 366,0 & 432,0 & 27,0 \\
\hline & $\mathrm{Tp}\left({ }^{\circ} \mathrm{C}\right)$ & 60,0 & 188,0 & 312,0 & 396,0 & 453,0 & 321,0 \\
\hline & Perda de massa (\%) & 8,5 & 1,8 & 47,2 & 15,8 & 5,7 & 78,5 \\
\hline \multirow[t]{3}{*}{$\mathrm{RNi}$} & $\mathrm{Ti}\left({ }^{\circ} \mathrm{C}\right)$ & 24,0 & 121,0 & 232,0 & 386,0 & 455,0 & 24,0 \\
\hline & $\mathrm{Tp}\left({ }^{\circ} \mathrm{C}\right)$ & 57,0 & 208,0 & 321,0 & 424,0 & 478,0 & 336,0 \\
\hline & Perda de massa (\%) & 7,6 & 3,0 & 38,0 & 12,5 & 11,4 & 72,4 \\
\hline \multirow[t]{3}{*}{$\mathrm{RCd}$} & $\mathrm{Ti}\left({ }^{\circ} \mathrm{C}\right)$ & 34,0 & 102,0 & 241,0 & 397,0 & 474,0 & 34,0 \\
\hline & $\mathrm{Tp}\left({ }^{\circ} \mathrm{C}\right)$ & 55,0 & 208,0 & 326,0 & 439,0 & 498,0 & 342,0 \\
\hline & Perda de massa (\%) & 5,9 & 4,0 & 39,4 & 14,4 & 9,5 & 72,8 \\
\hline \multirow[t]{3}{*}{$\mathrm{RCu}$} & $\mathrm{Ti}\left({ }^{\circ} \mathrm{C}\right)$ & 23,0 & 106,0 & 254,0 & 384,0 & 427,0 & 24,0 \\
\hline & $\mathrm{Tp}\left({ }^{\circ} \mathrm{C}\right)$ & 58,0 & 225,0 & 322,0 & 406,0 & 451,0 & 333,0 \\
\hline & Perda de massa (\%) & 6,3 & 6,1 & 39,8 & 9,2 & 12,8 & 74,5 \\
\hline \multirow[t]{3}{*}{$\mathrm{RCo}$} & $\mathrm{Ti}\left({ }^{\circ} \mathrm{C}\right)$ & 41,0 & 91,0 & 208,0 & 380,0 & 449,0 & 41,0 \\
\hline & $\mathrm{Tp}\left({ }^{\circ} \mathrm{C}\right)$ & 56,0 & 98,0 & 323,0 & 418,0 & 466,0 & 344,0 \\
\hline & Perda de massa (\%) & 5,2 & 5,6 & 39,5 & 15,2 & 10,2 & 71,0 \\
\hline
\end{tabular}

${ }^{*} \mathrm{Ti}$ - Temperatura inicio do evento; ${ }^{*} \mathrm{Tp}$ - Temperatura de pico do evento 
portamento linear da perda de massa com variação de 9,5 a $12,8 \%$ para a amostra $\mathrm{RCd}$ e $\mathrm{RCu}$, respectivamente. Com exceção para a amostra $\mathrm{RCr}$ que apresentou uma decomposição de $5,7 \%$. Esta decomposição foi maior nas amostras $\mathrm{RCu}$ e $\mathrm{RCd}$, pode ser decorrente da quebra de ligaçôes do carbono inorgânico presente na forma de composto carbonatados $\left(\mathrm{CO}_{3}{ }^{2-}\right.$, $\mathrm{HCO}_{3}^{-}$).

Mercê et al (2000) ao estudarem a utilização de soluções de um biopolímero natural como agente complexante dos íons metálicos $\left(\mathrm{Co}^{2+}, \mathrm{Mn}^{2+}, \mathrm{Ni}^{2+}\right.$ e $\mathrm{Zn}^{2+}$ ), obteve curvas de TG com comportamento térmico semelhante às curvas obtidas neste trabalho.

Otero et al (2002) quando estudaram a decomposição térmica de diferentes tipos de lodo oriundos de diferentes estações de tratamento de esgoto (urbano e industrial) através de curvas TG/DTG e DTA, observaram comportamento termoanalítico semelhante às amostras de RSO e RM estudado neste trabalho.

Mangrich et al (2000) ao pesquisarem a decomposição térmica e o comportamento cinético de vermicompostos, onde apresentaram picos de decomposição térmica nas faixas de temperaturas semelhantes aos encontrados por esta pesquisa.

\section{CONCLUSÃO}

As concentrações dos sólidos totais fixos nas amostras com metais foram maiores que a amostra RSO, devido à retenção dos metais pelo absorvente em sua superfície e confirmado pela análise de infravermelho e TG/DTG.

As bandas dos espectros na regiáo do infravermelho das amostras apresentaram características de grupos carbonilados, as amostras que sorveram os metais obtiveram bandas de vibração que demonstraram evidências de ligação química entre o metal e o absorvente, devido à presença de bandas de compostos carbonilados coordenados e existência da ligação metal-oxigênio.

As amostras submetidas ao processo de sorção apresentaram modificaçôes importantes no perfil da curva TG/DTG, alterando as temperaturas de decomposição do biossólido usado como absorvente dos metais, estas alterações foram confirmadas pelas variaçôes das concentraçōes dos parâmetros físicos e químicos (Umidade,
ST, STF, STV, COT, NTK e DQO)

das amostras.

O perfil das curvas TG/DTG apresentou modificaçôes significativas, tanto no âmbito qualitativo e quantitativo dos eventos, demonstrando que a sorção dos metais altera as características físicas e químicas do absorvente, aumentando a sua estabilidade quanto à decomposição.

\section{AGRADECIMENTOS}

Os autores agradecem o apoio financeiro concedido pelo $\mathrm{CNPq}$ e a CAPES para realização deste trabalho de pesquisa.

\section{REFERÊNCIAS}

APHA; AWWA; WEF. Standard methods for the examination of waterwaste. 19th ed. Washington. American Public Health Association, American Water Works Associations, Water Environmental Federation, p. 1134. 1995.

ARICAN, B.; GOKEAY, C. F. YETIS, U. Mechanistics of nickel sorption by activated sludge. Process Biochemistry. v. 37, p. 1307-1315, 2002.

ATKINS, P. W. Físico-quimica. $6^{\circ} \mathrm{Ed}$. vol 2. Rio de Janeiro. Ed. LTC, 382p. 1999.

BARROS, A. J. M. et al. III-094-Estudo termogravimétrico do lodo de esgoto sanitá rio usado no processo de biossorção de metais pesados. In: XI SIMPÓSIO LUSO-BRASILEIRO DE ENGENHARIA SANITÁRIA E AMBIENTAL - SILUBESA, Anais... Natal. ABESAPESB-APRH, CD-ROM, 2004.

BARROS JR. L. et al. Remoção de metais pesados presentes nas águas de produção de campos de petróleo. In: XV CONGRESSO BRASILEIRO DE ENGENHARIA QUÍMICA - COBEQ, 4 , Anais... Natal. ABEQ, CD-ROM. 2002.

BRANCO, S. M. Hidrobiologia aplicada à engenharia sanitária. $3^{\circ}$ ed. São Paulo. Ed. CETESB/ASCETESB, 616 p. 1986.

CLARISSE, M. D.; AMORIM, M. C. V.; LUCAS, E. F. Despoluição ambiental: uso de polímeros na remoção de metais pesados. Revista de Química Industrial. n. 715, p. 16-24, 1999.

ECCLES, H. Removal of heavy metals from effluent streams - why select a biological process? Journal Internacional Biodetermination Biodegradation, v. 44, n. 5, p. 5-16, 1995.

FELLENBERG, G. Introdução aos problemas da poluição ambiental. São Paulo. Ed. EPU. 196 p., 1980.

FERENC, W.; WALKÓW-DZIEWULSKA, A. 2,3-Dimethoxybenzoates of heavy lanthanides and yttrium. Journal of Thermal Analysis and Calorimetry. v. 71, p. 375-386, 2003.

GOMES, N. C. M.; MENDONÇA-HAGLER, L. C. S.; SAVVAIDIS, I. Metal bioremediation by microorganisms. Revista Microbiology. n. 29, v. 2, p. 85-92, 1998.

HATAKEYAMA, T.; QUINN, F. X. Thermal Analysis: fundamental and applications to polymer science. New York. Ed. John Wiley, 158 p., 1997.

MALLIKARJUNA, N. N., VENKATARAMAN, A. Adsorption of $\mathrm{Pb}^{2+}$ ions on nanosized -Fe2O3: formation of surface ternary complexes on ligand complexation. Talanta, v. 60, p. 139147, 2003.

MANGRICH, A. S. et al. Criterious preparation and characterization of earthworn-composts in view of animal waste recycling. Part I. correlation between chemical, thermal and FTIR spectroscopic analyses of four humic acids from earthworm-composted animal manure. Journal Brazilian Chemistry Society. 11 (2), p. 164-169. 2000.

MERCE, A. L. R. et al. Evaluation of the complexes of galactomannan of leucaena and $\mathrm{Co}^{2+}$, $\mathrm{Mn}^{2+}, \mathrm{Ni}^{2+}$ and $\mathrm{Zn}^{2+}$. Journal Brazilian Chemistry Society. 1 (3), p. 224-231. 2000.

METCALF AND EDDY. Wastewater engineering: treatment, disposal and reuse. 3rd ed. New York. Ed McGraw-Hill International. 1334 p., 1991.

MOTHÉ, C. G.; AZEVEDO, A. D. Análise térmica de materiais. São Paulo. Ed. Ieditora, p. 300, 2002.

OTERO, M. et al. Thermogravimetry as a technique for establishing the stabilization progress of sludge from wastewater treatment plants. Thermochemical Acta 389, p. 121-132. 2002.

PAIVA, D. L., LAMPMAN, G. M., KRIZ, G. S. Introduction to spectroscopy: a guide for students of organic chemistry. 2nd ed. New York. Harcourt Brace College Publishers, p 511., 1996.

PINTO, G. A. S. et al. Aplicação de microrganismos no tratamento de resíduos: a remoção de metais pesados de efluentes líquidos. In: www. estagio.br/methodus/5/capitulo09.htm Acesso em 06 dez 2001.

SAWYER, C. N.; MCCARTY, P.L.; PARKIN, G. F. Chemistry for environmental engineering. 4th ed. New York. Ed. McGraw-Hill. 658 p., 1994.

SOUZA, A. G. et al. A thermoanalytic and kinetic study of sunflower oil. Brazilian Journal of Chemical Engineering. v. 21, n. 2, p 265-273, 2004.

VALDMAN, E.; LEITE, S. G. F. Biosorption de $c d$, zn and $c u$ by Sargassum sp. waste biomass. Bioprocess Engineering. v. 22, p. 171-173, 2000.

VOLESKY, B. Biosorption of heavy metals. Montreal. Ed. CRC Press, McGill University, p. 396, 1989.

Endereço para correspondência:
Aldre Jorge Morais Barros Rua João Agripino dos Santos, 252 58 $106-000$ Campina Grande - PB- Brasil
'Tel: (83) 3339-7520
Fax: (83) 3310-1 II4
E-mail: ajmbarros@yahoo.com.br 